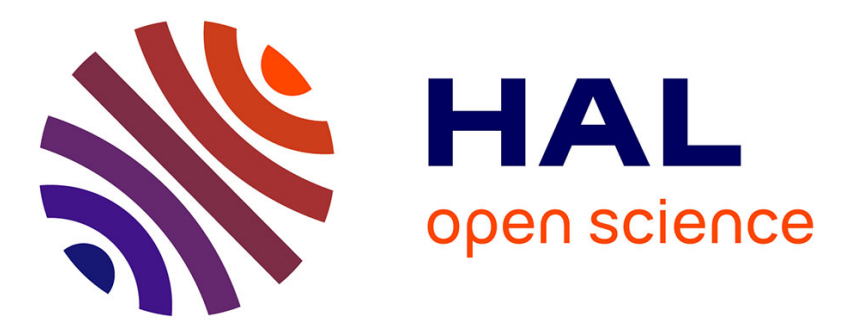

\title{
Silicon germanium on graded buffer as a new platform for optical interconnects on silicon
}

\author{
Vladyslav Vakarin, Papichaya Chaisakul, Jacopo Frigerio, Andrea Ballabio, \\ Xavier Le Roux, Jean-René Coudevylle, Laurent Viviven, Giovanni Isella, \\ Delphine Marris-Morini
}

\section{To cite this version:}

Vladyslav Vakarin, Papichaya Chaisakul, Jacopo Frigerio, Andrea Ballabio, Xavier Le Roux, et al.. Silicon germanium on graded buffer as a new platform for optical interconnects on silicon. SPIE Photonics West, Feb 2016, San Francisco, CA, United States. 10.1117/12.2208661 . hal-01322066

\section{HAL Id: hal-01322066 https://hal.science/hal-01322066}

Submitted on 30 May 2016

HAL is a multi-disciplinary open access archive for the deposit and dissemination of scientific research documents, whether they are published or not. The documents may come from teaching and research institutions in France or abroad, or from public or private research centers.
L'archive ouverte pluridisciplinaire HAL, est destinée au dépôt et à la diffusion de documents scientifiques de niveau recherche, publiés ou non, émanant des établissements d'enseignement et de recherche français ou étrangers, des laboratoires publics ou privés. 


\title{
Silicon germanium on graded buffer as a new platform for optical interconnects on silicon
}

\author{
Vladyslav Vakarin* ${ }^{\mathrm{a}}$, Papichaya Chaisakul ${ }^{\mathrm{a}, \mathrm{b}}$, Jacopo Frigerio ${ }^{\mathrm{c}}$, Andrea Ballabio $^{\mathrm{c}}$, Xavier Le Roux $^{\mathrm{a}}$, \\ Jean Rene Coudevylle ${ }^{\mathrm{a}}$, Laurent Viviven ${ }^{\mathrm{a}}$, Giovanni Isella ${ }^{\mathrm{c}}$, and Delphine Marris-Morini ${ }^{\mathrm{a}}$ \\ anstitut d'Electronique Fondamentale,Univ.Paris Sud, CNRS,Univ.Paris Saclay,UMR 8622,Bât. \\ 220, 91405 Orsay Cedex, France; \\ ${ }^{\mathrm{b}}$ Departement of Materials Engineering, The University of Tokyo, 7-3-1 Hongo, Bunkyo-Ku, Tokyo; \\ ${ }^{c}$ L-NESS Dipartimento di Fisica del Politecnico di Milano, Como, Italy
}

\begin{abstract}
We experimentally and theoretically investigate the use of silicon germanium ( $\mathrm{SiGe}$ ) on silicon substrate as a new platform for optical interconnects. The system composed of Germanium $(\mathrm{Ge})$ rich $\mathrm{Si}_{1-\mathrm{x}} \mathrm{Ge}_{\mathrm{x}}$ guiding layer on a graded SiGe layer is showed to be suitable for the realization of all main building blocks of passive optical circuitry. We show experimentally at a wavelength of $1550 \mathrm{~nm}$ that sharp $12 \mu \mathrm{m}$ radius bends can be obtained by light confinement tuning. Mach-Zehnder interferometer with more than $10 \mathrm{~dB}$ extinction ratio is also demonstrated. Moreover, Ge-rich $\mathrm{Si}_{1-\mathrm{x}} \mathrm{Ge}_{\mathrm{x}}$ based passive components are very interesting for their native integration with Ge-rich active optical devices. Hence, by using this new platform for optical integrated circuits, lattice mismatch between silicon and germanium is no longer a major constraint for the integration of Ge-rich active photonic components on silicon.
\end{abstract}

Keywords: Silicon photonics, Germanium, Waveguide, Bends, Mach-Zehnder interferometer

\section{INTRODUCTION}

Continuously increasing data consumption pushes electronic integrated circuits to their limits. To tackle high power consumption issue and bandwidth limitations, optical interconnects may possibly be the most viable solution. In this context, photonic platform material must be compatible with mature silicon ( $\mathrm{Si}$ ) based technology. Hence, Germanium is identified as a promising candidate for Si compatible photonic platform. Germanium, for its pseudo-direct band gap properties was widely studied for the realization of laser sources ${ }^{1-2}$. Additionally, Germanium based modulators ${ }^{3-6}$ and photodetectors $^{7}$ are remarkable for their speed and power consumption. However, the integration of these devices with passive circuitry is challenging. As $\mathrm{Si}$ and Ge have 4\% lattice mismatch, straightforward integration of high quality active devices is difficult due to high dislocation rate. An efficient strategy to accommodate Si and Ge lattice parameters is the use of a graded buffer layer. We recently proposed that such graded buffer layer can be used to confine light in a Ge-rich SiGe virtual substrate and obtain SiGe waveguide on bulk silicon. Such approach makes high Ge content photonic components integration direct: high quality bulk $\mathrm{Ge}$ or $\mathrm{Ge} / \mathrm{SiGe}$ multi-quantum well structures can be grown on $\mathrm{Si}_{1-\mathrm{x}} \mathrm{Ge}_{\mathrm{x}}$ (with $\mathrm{x}$ higher then 0.8) guiding layer.An optical link composed of a Ge/SiGe quantum well (QW) photodetector and modulator connected by $\mathrm{Si}_{0.16} \mathrm{Ge}_{0.84}$ waveguide has thus been demonstrated ${ }^{8}$. Until now only straight waveguides were demonstrated with this approach and compact guiding structures were still a challenge due to the low index contrast in these waveguides. We experimentally show that Ge rich SiGe on graded buffer waveguides are a promising platform for optical interconnects on silicon. Sharp bends and Mach Zehnder interferometers are demonstrated. Hence Ge-rich SiGe optical circuitry paves the way for the monolithic integration of Ge based active devices on silicon.

* vladyslav.vakarin@u-psud.fr 


\section{EPITAXIAL GROWTH, DESIGN AND FABRICATION}

\subsection{SIGE GROWTH}

The structure was grown by low energy plasma enhanced chemical vapor deposition (LEPECVD). The $\mathrm{Si}_{1-\mathrm{y}} \mathrm{Ge}_{\mathrm{y}}$ graded buffer is grown on a $100 \mathrm{~mm} \mathrm{Si}$ substrate with a deposition rate of 5-10 nm/s. Ge concentration y is linearly increased from 0 to $79 \%$ during the growth over $11 \mu \mathrm{m}$ thickness which correspond to a refractive index variation from 3.477 to 4.112 at $1550 \mathrm{~nm}$ wavelength. Then, $2 \mu \mathrm{m}$ thick relaxed $\mathrm{Si}_{0.2} \mathrm{Ge}_{0.8}$ guiding layer is grown on the top of the graded buffer. A difference of $1 \%$ in Ge concentration between the top of the graded buffer and the guiding layer does not affect the material quality and provides better vertical light confinement. A cross section of sample layers is shown in Fig.1. It is important to stress that this stack can be used for fabrication of passive circuitry and can also act as a substrate on which quantum wells with well-chosen thickness and germanium content surrounded by doped regions can be deposited by low temperature $\left(\leq 450^{\circ} \mathrm{C}\right)$ epitaxial growth.

\section{$2 \mu \mathrm{m} \mathrm{Si} \mathrm{i}_{0.2} \mathrm{Ge}_{0.8}$}

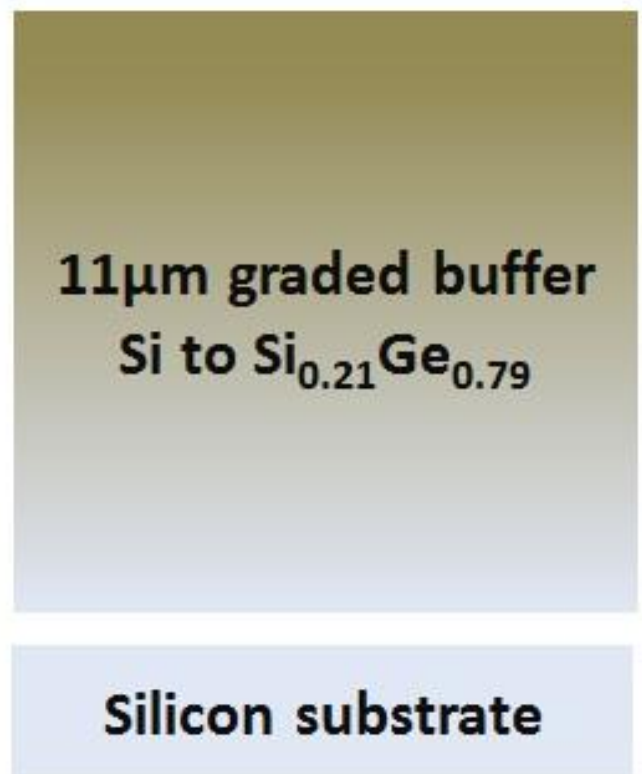

Figure 1. Schematic diagram of Ge-rich $\mathrm{SiGe}$ waveguide 


\subsection{PASSIVE DEVICES DESIGN AND FABRICATION}

To obtain sharp and compact footprint $90^{\circ}$ bends two kind of waveguides were chosen: $2 \mu \mathrm{m}$ wide and $1 \mu \mathrm{m}$ etched (Fig.2(a)) for straight portions and $2 \mu \mathrm{m}$ wide and $2.5 \mu \mathrm{m}$ etched (Fig.2(b)) for bent portions.
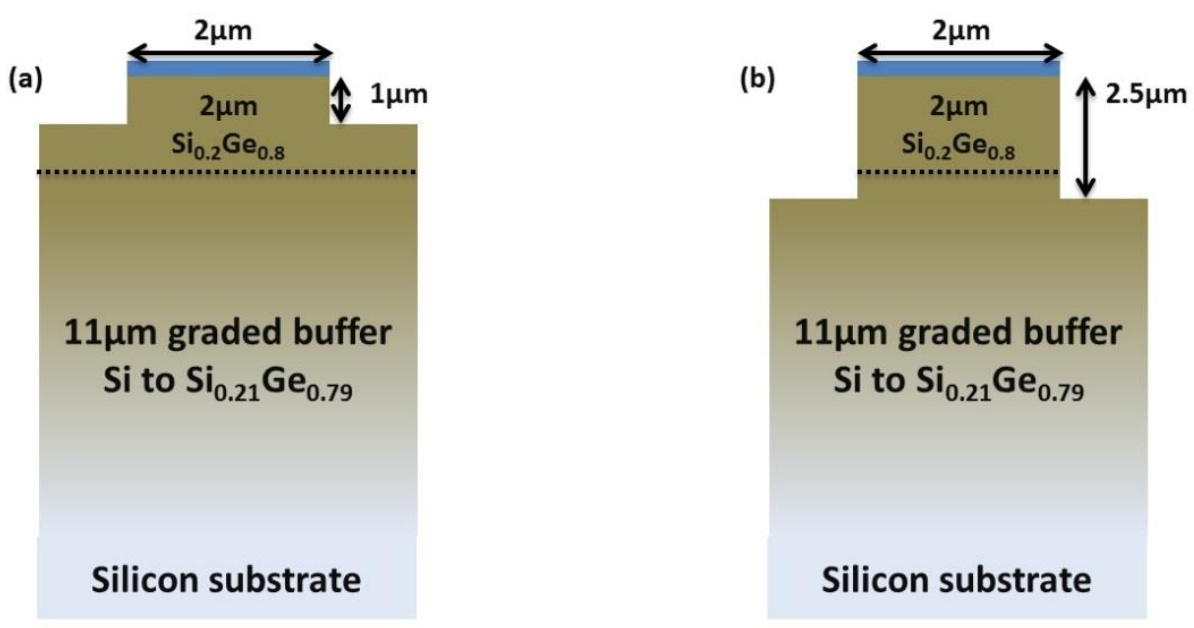

$200 \mathrm{~nm} \mathrm{SiO}$

Figure 2. (a) Slightly etched waveguide used for straight waveguide portions. (b) Deeply etched waveguide for bent portions

Slightly etched waveguides, with their low confinement, as shown in Fig.3(a), are used for their low propagation losses. By deep etching bent waveguide sections (Fig.3(b)) we reduce radiation losses of the bends ${ }^{9}$. Thus sharper bends can be achieved with light confinement tuning. Using only slightly etched rib waveguides it is impossible to obtain bends with radii below $200 \mu \mathrm{m}$ with acceptable losses for telecom wavelengths.

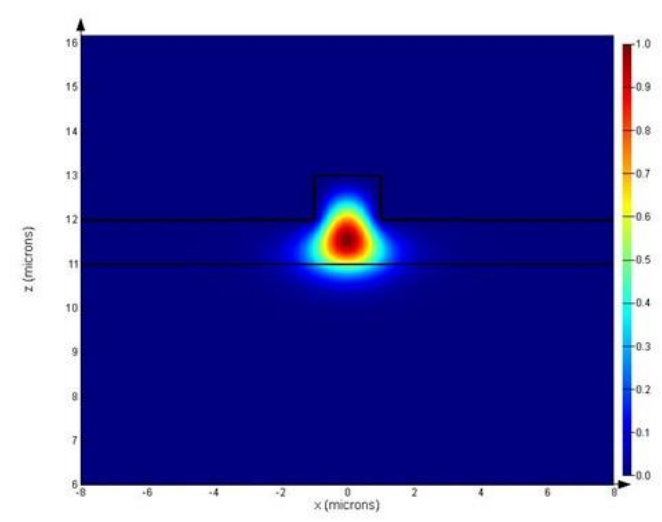

(a)

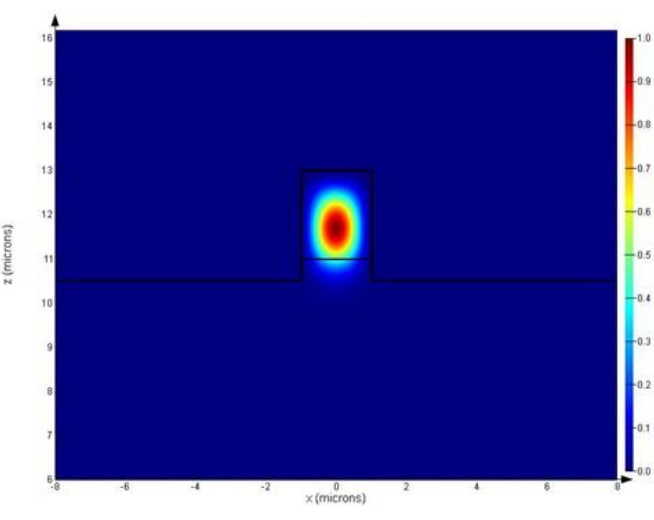

(b)

Figure 3. (a) Slightly etched waveguide field intensity profile in TE polarization. (b) Deeply etched waveguide field intensity profile in TE polarization 
Based on the growth reported on Figure 1, sharp bends and asymmetric Mach-Zehnder interferometers were fabricated. As a first step, $200 \mathrm{~nm}$ silicon dioxide $\left(\mathrm{SiO}_{2}\right)$, was deposited on the top of the waveguide layer. Then, $\mathrm{SiO}_{2}$ hard mask was fabricated by deep UV lithography and inductively coupled plasma etching (ICP). Rib waveguides were then defined by ICP etching (Fig2. (a)). Finally $90^{\circ}$ bends and bent portions of Mach Zehnder interferometers were deeply etched using 200nm $\mathrm{SiO}_{2}$ hard mask (Fig4.(a) and (b)). Silicon dioxide hard mask is used for a selfalignement of slightly and deeply etched waveguides. After each ICP etch, hydrogen peroxide solution $\left(\mathrm{H}_{2} \mathrm{O}_{2}\right)$ was used to smooth sidewall roughness

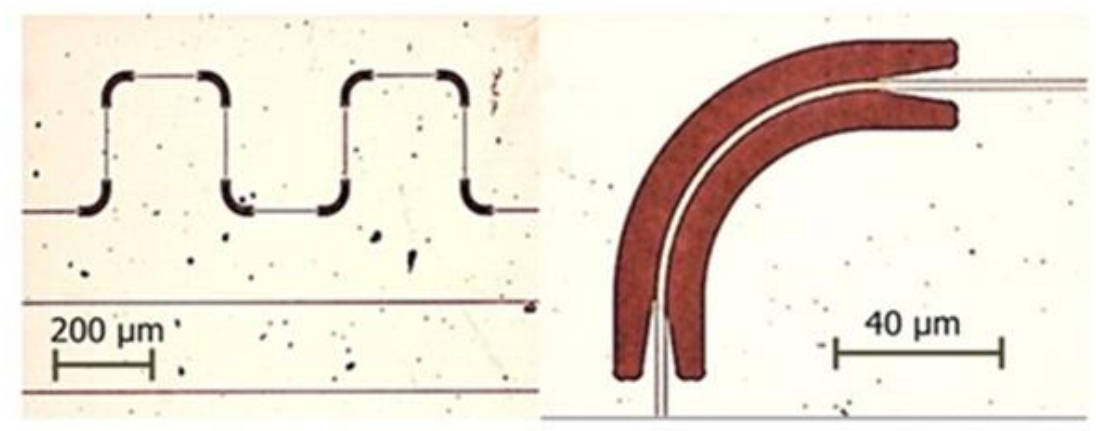

(a)

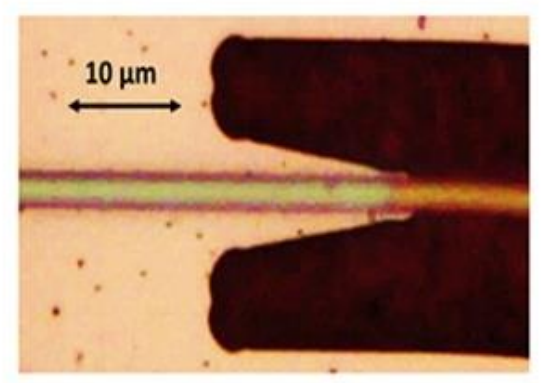

(c) (b)

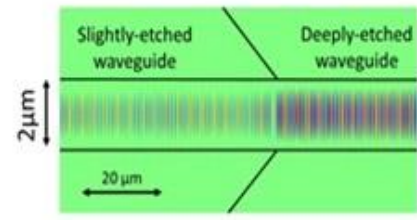

top view

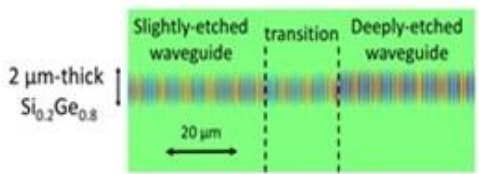

side view

\section{(d)}

Figure 4. (a) Optical microscope view of fabricated bends. (b) Zoom on a $90^{\circ}$ bend, deeply etched region appear darker. (c) Zoom on the transition between slightly and deeply etched waveguide regions (d) propagation simulation for the transition section calculated in TE polarization with eigen mode expansion solver

A transition is needed between differently etched portions of the bend to avoid an abrupt change in the effective index. Thus, to reduce transition losses, a tapered $20 \mu \mathrm{m}$ long transition as in Fig. 4(c) was designed. Numerical simulations were performed for the design of this transition and the results are reported in Fig. 4(d). For the side view, a slice located $1 \mu \mathrm{m}$ below the top surface of the waveguide is considered for the calculation while a simulation is performed on the slice located in the middle of the waveguide for the side view. From simulations no radiation losses originating from the transition between the two waveguides is confirmed. 
It is critical, for a photonic platform to be able to tune the phase of one wave with respect to another one. In this way, Mach-Zehnder interferometers based on $\mathrm{SiGe}$ waveguides were investigated to prove the validity of the chosen SiGe on graded buffer photonic platform. In this objective Multi-Mode beam splitters have been designed, based on the slightly etched waveguide reported in Fig.2 (a). Parameter optimization gave a $187 \mu \mathrm{m}$ long, $11 \mu \mathrm{m}$ wide beam splitter with a $2 \mu \mathrm{m}$ width input waveguide and $3.5 \mu \mathrm{m}$ width output waveguides separated by $2.3 \mu \mathrm{m}$. In Fig. 5 (a) and (b) are shown the schematic view of the optimized beam splitter and the corresponding propagation simulation. Using this type of beam splitter, an asymmetric Mach-Zehnder was designed as shown in Fig.5(c).
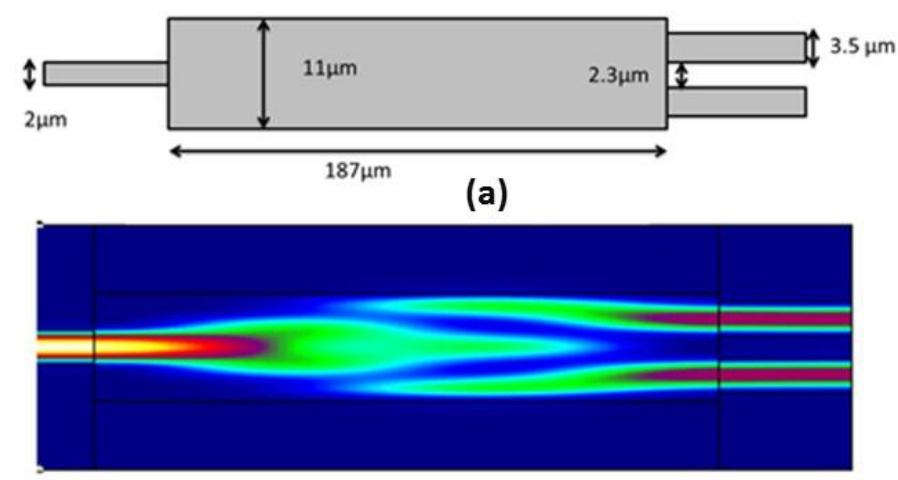

(b)

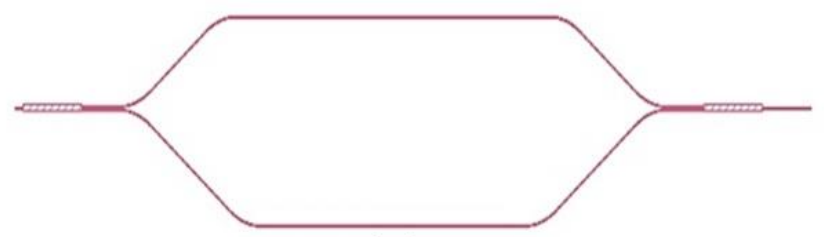

(c)

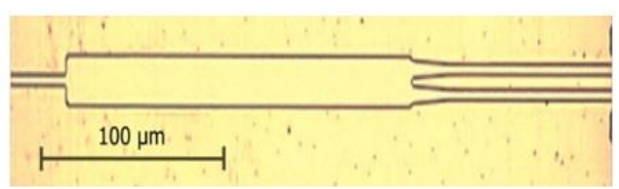

(d)

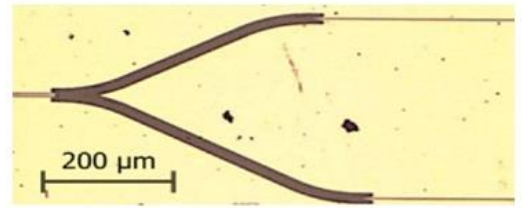

(e)

Figure 5. (a) Optimized design for 1x2 MMI beam splitter. (b) Electric field's component propagation simulation. (c) asymmetric Mach Zehner interferometer general schematic view. (d) Zoom on fabricated beam splitter under optical microscope. (e) view of the deeply etched region (they appear darker) in the bent region of the Mach Zehnder interferometer

Fig 5.(d) and (e) shows a zoom under an optical microscope on the different parts of the fabricated Mach-Zehnder: MMI beam splitter and bent parts using deeply etched waveguide to separate both arms. 


\section{III.PASSIVE STRUCTURE CARACTERISATION AND DISCUSSION \\ 3.1 BENDS CARACTERISATION}

Fabricated $90^{\circ} \mathrm{SiGe}$ were characterized using a butt coupling technique: TE polarized light from a tunable laser was injected in the waveguide with a lensed fiber, an objective collected the output light and injected it to a photodetector. $12,25,50$ and $100 \mu \mathrm{m}$ radii were studied. To obtain reliable bend loss data a set of 32 consecutive bends for each bend radius were fabricated. Measurements were performed at $1550 \mathrm{~nm}$ which is above the indirect optical absorption bandedge for $\mathrm{Si}_{0.2} \mathrm{Ge}_{0.8}$

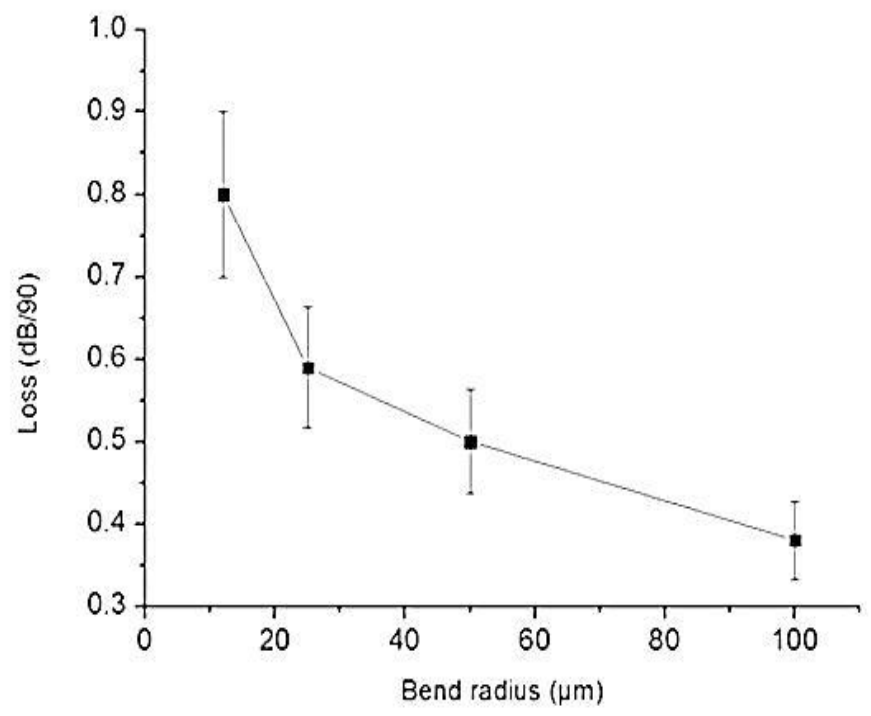

Figure 6. (c) Measured losses of the $90^{\circ}$ bends as function of bend radius

Straight waveguide losses were estimated to be $1 \mathrm{~dB} / \mathrm{mm}$. Propagation losses can be reduced by optimizing both surface passivation and etching process to reduce sidewall roughness. In Fig.6(c) are reported $90^{\circ}$ bend losses as a function different bend radii. For $50 \mu \mathrm{m}$ bend radius, $0.5 \mathrm{~dB} / 90^{\circ}$ losses were obtained. Numerical calculations show that the main contribution for the bend losses is the modal mismatch between straight and bent section of a $90^{\circ}$ bend. $0.1 \mathrm{~dB} / 90^{\circ}$ can further be obtained by using an offset technique ${ }^{9}$ or by continuously varying bend curvature ${ }^{10}$. 


\subsection{MACH ZEHNER INTERFEROMETERS}

Asymmetric Mach Zehnder interferometers were characterized by the same butt coupling method. As reported in Fig.7, more than $10 \mathrm{~dB}$ extinction ratio is obtained. The measured free spectral range is $24.4 \mathrm{~nm}$ and it matches theoretical expectations for a $24 \mu \mathrm{m}$ arm difference.

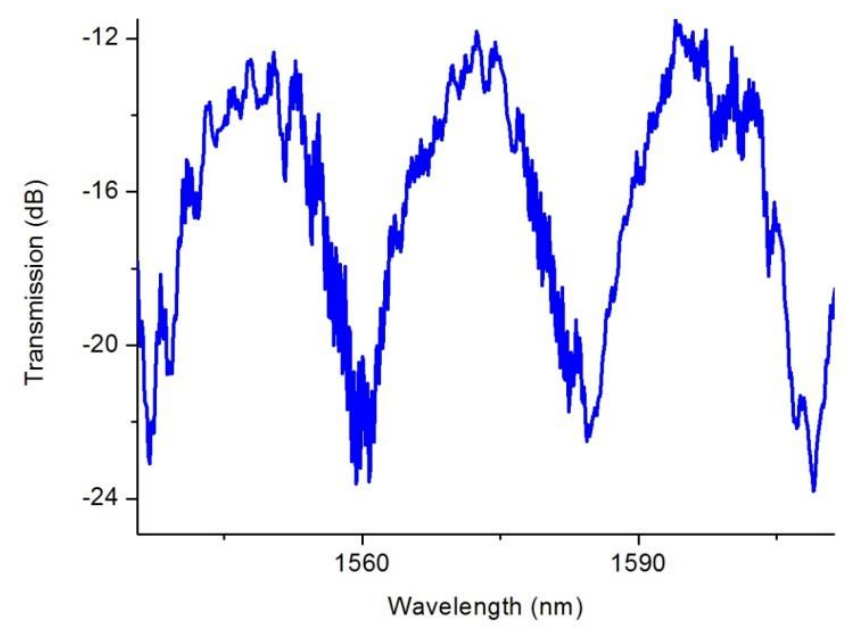

Figure7. Optical transmission of the fabricated asymmetric Mach-Zehnder interferometer. 24.4nm free spectral range is corresponding to the length difference of $24 \mu \mathrm{m}$ between both arms of the Mach-Zehnder

Between the input and output 12dB losses were estimated. This includes: $8 \mathrm{~dB}$ losses for $8 \mathrm{~mm}$ total length of access waveguides i.e input and output, $2 \mathrm{~dB}$ loss in Mach-Zehnder arms and $1 \mathrm{~dB}$ losses for each coupler. The length of access waveguides can be decreased, thus dramatically reducing their contribution to the total losses.

\section{CONCLUSIONS}

Ge-rich silicon-germanium on graded buffer offers a new possibility of integration of Ge based active and passive devices on bulk silicon substrate. The high Ge content of the proposed passive circuitry allows the growth of high quality $\mathrm{Si}_{1-\mathrm{x}} \mathrm{Ge}_{\mathrm{x}}$ layers for active devices. Main building blocks such as sharp and compact footprint $90^{\circ}$ bends and MachZehnder interferometers with good extinction ratio have been designed and experimentally validated. Light confinement tuning was used to develop compact passive circuitry and reduce radiation losses. Perfect alignment during the fabrication was achieved by self-aligned two etching process to use both slightly and deeply etched region. This work paves the way for the demonstration of wavelength division multiplexing devices. Their further integration with light sources, modulators, photodetectors will lead us to SiGe based photonic integrated transceivers on silicon substrate.

\section{ACKNOWLEDGEMENTS}

The CAPRILO foundation is acknowledged for financial support through the grant EIDOS 2011-038. The fabrication of the device was performed at the CTU-IEF-MINERVE nano-center, which is partially funded by "Conseil Général de l'Essonne". This work was partly supported by the French RENATECH network. D.M.M acknowledges support by Institut Universitaire de France. Marie Curie International Outgoing Fellowships through grant agreement PIOF-GA2013-629292 MIDEX is partly acknowledged. This project has received funding from the European Research Council (ERC) under the European Union's Horizon 2020 research and innovation program (grant agreement $\mathrm{N}^{\circ} 639107-$ INSPIRE) 


\section{REFERENCES}

[1] Camaco-Aguilera, R.E., Cai,Y., Patel, N.,Besette, J.T., Romagnoli, M., Kimerling, L.C., and Michel, J., “An electrically pumped germanium laser," Opt.Express 20(10),11316-11320(2012).

[2] Koerner, R., Oehme, M., Gollhofer, M., Schmid, M., Kostecki, K., Bechler, S., Widmann, D., Kasper, E., and Schulze, J., "Electrically pumped lasing from Ge Fabry-Perot resonators on Si," Opt.Express 23(11), 14815$14822(2015)$.

[3] Gupta, S., Srinivasan, S. A., Pantouvaki, M., Chen, H., Verheyen, P., Lepage, G., Thourhout, D. V., Roelkens, G., Saraswat, K., Absil, P., and Van Campenhout, J., "50GHz Ge Waveguide Electro-Absorption Modulator Integrated in a 220nm SOI Photonics Platform," Optical Fiber Communication Conference 2015.

[4] Liu, J., Beals, M., Pomerene, A., Bernardis, S., Sun, R., Cheng, J., Kimerling, L.C., and Michel, J., "Waveguide-integrated, ultralow-energy GeSi electro-absorption modulators," Nat.Photonics 2(7), 433-437 (2008).

[5] Krishnamoorthy, A. V., Zheng, X., Feng, D., Lexau, J., Buckwalter, J. F., Thacker, H. D., Liu, F., Luo, Y., Chang, E., Amberg, P., Shubin, I., Djordjevic, S. S., Lee, J. H., Lin, S., Liang, H., Abed, A., Shafiiha, R., Raj, K., Ho, R., Asghari, M., and Cunningham, J. E., "A low-power, high-speed, 9-channel germanium-silicon electro-absorption modulator array integrated with digital CMOS driver and wavelength multiplexer," Opt. Express 22(10), 12289-12295 (2014).

[6] Chaisakul, P., Marris-Morini, D., Rouifed, M. S., Isella, G., Chrastina, D., Frigerio, J., Le Roux, X., Edmond, S., Coudevylle, J.-R., and Vivien, L., " $23 \mathrm{GHz}$ Ge/SiGe multiple quantum well electro-absorption modulator," Opt. Express 20(3), 3219-3224 (2012).

[7] Virot, L., Crozat, P., Fédéli, J.-M., Hartmann, J.-M., Marris-Morini, D., Cassan, E., Boeuf, F., and Vivien, L., "Germanium avalanche receiver for low power interconnects," Nat. Commun. 5, 4957 (2014).

[8] Chaisakul, P., Marris-Morini, D., Frigerio, J., Chrastina, D., Rouifed, M. S., Cecchi, S., Crozat, P., Isella, G., and Vivien, L., "Integrated germanium optical interconnects on silicon substrates," Nat. Photonics 8(6), 482488 (2014).

[9] Wada, K., Popovic, M., Akiyama, S., Haus, H. A., and Michel, J., "Micron-size bending radii in silica-based waveguides," Advanced Semiconductor Lasers and Applications/ Ultraviolet and Blue Lasers and Their Applications /Ultralong Haul DWDM Transmission and Networking/WDM Components, 2001. Digest of the LEOS Summer Topical Meetings, (Copper Mountain, CO USA, 2001), 13-14.

[10] Cherchi, M., Ylinen, S., Harjanne, M., Kapulainen, M., and Aalto, T., "Dramatic size reduction of waveguide bends on a micron-scale silicon photonic platform," Opt. Express 21, 17814-17823 (2013). 